\title{
Particle Swarm Optimization of Air-cored Axial Flux Permanent Magnet Generator for Small-Scale Wind Power Systems
}

\author{
B. Xia, P. C. K. Luk, W. Fei, L. Yu \\ Electric Power and Drive Group, School of Engineering, Cranfield University, UK
}

Keywords: Air-cored, axial flux permanent magnet, particle swarm optimization, wind power.

\begin{abstract}
Axial flux permanent magnet synchronous machines with aircored configuration is particular suitable for small scale wind power system due to their advantages of low synchronous reactance, cogging torque free, high efficiency and high power factor. However, due to the number of machine parameters, with some tightly 'coupled' with each other, optimisation of the design could become extremely challenging by conventional analytical means. Here, the particle swarm optimization method is used in the design of an axial flux permanent magnet generator for small-scale wind power system. Five inter-dependent design parameters are adjusted simultaneously to achieve an optimal solution for the application. Three-dimensional finite element analysis is employed to evaluate the electromagnetic performance for the optimization. The results show the proposed optimization method is efficient and with fast convergence.
\end{abstract}

\section{Introduction}

Wind power will continue to contribute an increasingly important fraction of the future energy mix in the U.K. Among the most mature technology in renewable energy industry, wind power has promising prospects to achieve an economically sustainable and environment friendly solution to energy challenge we face $[1,2]$. Whilst large-scale wind generators are now widely employed in centralized wind farms to provide electricity to the grid [2,3], the fast development of distributed electric generation means that the demand for small-scale to medium-scale generators will remain strong. In particular, for some cities and towns in windy area, small-scale wind generators can be built in neighbourhoods and even clustered together to power streetlights. Moreover, in some remote areas where power grid is not available, small-scale off-grid power systems become more crucial as the main energy source $[4,5]$.

With the advantages of flat shape, compact structure, high power and torque density, axial flux permanent magnet (AFPM) synchronous machines are widely applied in various applications such as wind power generation, electric vehicle, aircraft and so on [6-8]. Air-cored AFPM synchronous machines, which have no ferromagnetic material in the stator, could eliminate iron loss as well as cogging torque. Moreover, such an air-cored configuration could also deliver high efficiency, light weight and low starting torque so that it will be ideal for small-scale off-grid wind power applications. As the air-cored AFPM synchronous machine has relatively large magnetic air gap, more magnets are required for the sufficient excitation. Nevertheless, the advantage of large air gap is that no saturation occurs in the machine, and high harmonic components of air gap flux density distribution can be minimized. Importantly, the large effective air gap length also results in low synchronous reactance and hence high power factor.

The electromagnetic performance of an AFPM generator is affected by many parameters, and some of them have strong interactions with one another. This could present conflicting requirements in the optimisation stage. Many optimization methods, such as response surface methodology (RSM), genetic algorithm (GA) and particle swarm optimization (PSO), have been proposed for multi-parameter and multiobjective problems to explore the design space and achieve optimal designs to satisfy the objectives simultaneously [911]. Among these methods, PSO is a relatively simple but efficient evolutionary computation technique originated from the movement around searching space of intelligent swarm societies according to a user-defined fitness function. The instinct merits such as simple parameter adjustment, short computational time, derivative-free, and flexibility, make PSO easily suit to different kinds of optimization problems [11-13]. Although it was only first developed in 1995 [14,15], PSO has already attracted more and more interests in a variety of research areas and applications.

PSO method has been adopted successfully in electric power systems for economic dispatch, reactive power control and voltage regulations $[10,12,16]$. In the recent years, increasing number of researchers are applying PSO method to multiparameter, multi-objective optimal design of electric machines, as many of the parameters to be optimized are conflicting to each other during optimization. Permanent magnet synchronous machines (PMSMs) have been optimized using PSO to achieve high efficiency and less weight $[17,18]$. Moreover, the cogging torque of a transverse flux permanent motor (TFPM) and the force of a linear TFPM have been optimized in [9] and [19] respectively. An PSObased optimal design of axial laminated synchronous reluctance motor (SynRM) has been used for traction applications [20]. Moreover, designs of induction machines using PSO method have also been reported [21].

This paper concerns the design of a 500W AFPM generator for small-scale wind power system. Though with many inherent merits of this air-cored AFPM synchronous machine, 


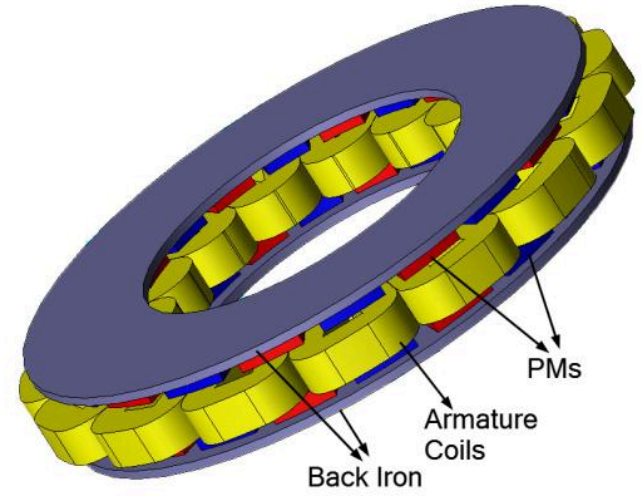

Fig. 1: Topology of air-cored AFPM machine.

there is a concern about the usage of permanent magnet (PM) materials hence the cost of the machine. Therefore, the inner and outer diameters, pole pitch factor and thickness of PMs should be carefully determined to obtain high power output with minimum PM usage. Moreover, since no slot exists in the stator, the coil configuration, which has a great influence on the performance of the generator, needs to be optimized. Three-dimensional (3-D) finite element analysis (FEA) models are developed to carry out the optimization based on PSO. Optimal design is achieved by factoring both the performance and cost into the cost function. The electromagnetic performance of the optimal design is evaluated by 3-D FEA under different operational conditions. The results have revealed that PSO is a simple yet efficient method with fast convergence.

\section{The AFPM generator}

The air-cored AFPM synchronous machine with doubleouter-rotor-internal-stator configuration is illustrated in Fig. 1. Surface-mounted PM poles have the merits of simple structure and easy manufacture and assembly. The wind turbine can be directly attached to the outer rotor which can further reduce the system weight and cost. Since the gearbox is no longer necessary, efficiency and reliability will further improve [6]. Due to the relatively large magnetic air gap, saturation is not considered in analysis. Therefore the main design parameters lie on the dimensions of PM and coils.

According to its instinct flux distribution style, the sizing equation of this AFPM synchronous machine can be obtained

$$
P_{e m}=\frac{\pi^{2}}{120} m n \alpha_{p} K_{w} B_{g} A\left(1-\gamma^{2}\right)(1+\gamma) D_{o}^{3}
$$

where $P_{\mathrm{em}}$ is the electric power, $\alpha_{p}$ is pole pitch factor, $m$ is the number of phases, $n$ is rotating speed in resolution per minute, $K_{w}$ is coil factor, $B_{g}$ is the air gap flux density, $A$ is the electric load, $D_{o}$ is the outer diameter, and $\gamma$ is the ratio of $D_{i}$ (inner diameter) over $D_{o}$. Outer diameter is chosen as $250 \mathrm{~mm}$ to meet the requirements.

The combinations of rotor poles and stator coils for such AFPM machine have been discussed in [22]. The pole/coil configurations of $16 / 12,20 / 15$ and $24 / 18$ have large coil factors and the power density of the machine with increase with pole number. However, high pole number means strict precision for pole location, which will increase mechanical complexity and manufacturing cost. Therefore, a compromise of 20/15 pole/coil configuration is adopted for the design.

The main active parts in the machine are PMs, coils and back iron, as depicted in Fig.1. Since saturation in back iron is not considered, the size and shape of PMs and coils are the key parameters which have great influences on the performance of the machine. However, the design values of these parameters, such as pole pitch factor $\left(\alpha_{p}\right)$, inner diameter $\left(D_{i}\right)$, thickness of PM $\left(H_{m}\right)$, thickness of coil $\left(H_{c}\right)$ and coil-band width $\left(W_{c}\right)$, have great interaction, sometimes conflicting influence on one other. Consequently, they should be simultaneously optimized to achieve an optimal solution.

\section{Application of PSO}

PSO is a stochastic global optimization method. Besides its fast convergence rates, another main advantage is simplicity. Only two vectors are associated for each particle in PSO, position $\left(X_{i}\right)$ and velocity $\left(v_{i}\right)$ respectively. One position of a particle is a candidate solution, and the next searching position is determined by the velocity of the particle based on the experience of the whole swarm. After one iteration, position and velocity can be updated as follows [11]

$$
\begin{aligned}
& X_{i}(t+1)=X_{i}(t)+v_{i}(t+1) \\
& \begin{aligned}
v_{i}(t+1) & =w \cdot v_{i}(t)+c_{1} \cdot \operatorname{rand}() \cdot\left(p_{\text {best }}-X_{i}\right) \\
& +c_{2} \cdot \operatorname{rand}() \cdot\left(g_{\text {best }}-X_{i}\right)
\end{aligned}
\end{aligned}
$$

where $w$ is the initial weight, $c_{1}$ and $c_{2}$ are scaling factors, rand() is function generating random numbers between 0 and 1 , and $p_{\text {best }}$ is best solution one particle ever achieved (personal pest), and $g_{\text {best }}$ is the best solution all particle achieved so far (global best).

Much work has been done to achieve fast convergence on the selection of $w, c_{1}$ and $c_{2}$. According to [11,15], best choice for $c_{1}$ and $c_{2}$ is 2.0 for both, and $w$ should be between 0.4 and 0.9 .

\subsection{Define solution space}

Generally in engineering applications, parameters are chosen in a certain range based on practical experience and theories. In PSO, particles are exploring the solution space for optimal solution. Larger space means more time is required to explore the area. Thus reasonable range should be given according to different problem to save time for the optimization. For the AFPM machine design, there are five key design parameters having great influence on the machine performances:

- $\quad$ Ratio of $D_{i}$ over $D_{o}(\gamma)$ : Smaller $D_{i}$ is, longer the effective length of conductors are. Yet smaller $D_{i}$ reduces the space for armatures in inner radius area.

$$
\gamma \in(0.4,0.8)
$$

- $\quad$ Pole pitch factor $\left(\alpha_{p}\right)$ : Larger pole pitch not only can increase the flux density in the air gap, but also increase the amount of magnet usage and may cause unwanted harmonics in flux distribution. 


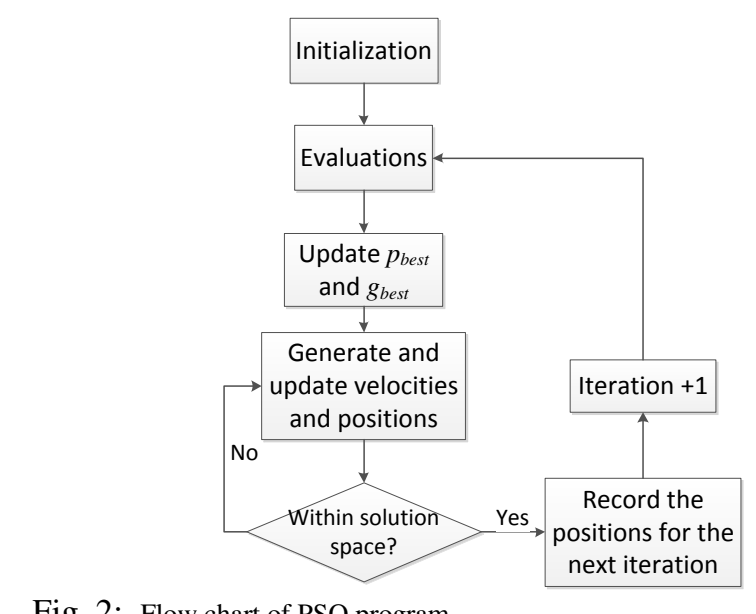

Fig. 2: Flow chart of PSO program.

\begin{tabular}{|l|l|l|l|l|l|l|l|}
\hline$w$ & $c_{1}$ & $c_{2}$ & $k_{m}$ & $k_{c}$ & $k_{v}$ & $k_{\eta}$ & $k_{p o}$ \\
\hline 0.65 & 2.0 & 2.0 & 30.0 & 2.0 & 1000 & 150 & 0.15 \\
\hline
\end{tabular}

Table 1: Values of factors and coefficients for PSO

$$
\alpha_{p} \in(0.5,1)
$$

- Thickness of magnet $\left(H_{m}\right)$ : Thicker magnet will increase the flux density as well as cost PM material.

$$
H_{m} \in(2,12)
$$

- $\quad$ Ratio of $H_{c}$ over $H_{m}(\beta)$ : Thicker coil means more conductors can be used to generate higher power. Nevertheless, thicker coils also enlarge the air gap and lower the flux density.

$$
\beta \in(0.5,2)
$$

- $\quad$ Ratio of $W_{c}$ over $R_{i}(\delta)$ : Wider coil-band can contain more conductors to generate higher torque, but more conductors also can cause problems such as higher armature resistance, higher loss, and thermal issues.

$$
\delta \in(0, \pi / 15)
$$

Population size also necessitates special attention. Large population can explore solution space more thoroughly, with the cost of more time-consuming computations. However, small population size of 10 to 20 can also provide sufficient exploration through the solution space and effectively reduce the optimization time [11]. Since the 3-D FEA evaluation is quite time-consuming, 10 particles are set for this case.

\subsection{Boundary conditions}

As there are random functions acting on the process of generating velocity, particles are likely to fly out of the defined solution space. In order to address this problem, boundary conditions are necessary to confine the particles. Three boundary conditions are normally employed for PSO, namely absorbing walls, reflecting walls and invisible walls. In the first two boundary conditions, particles are strictly restrained inside the solution space, while in invisible walls particles are allowed to go out of defined space. However, once a particle flies out, the position would not be calculated or evaluated, and the particle has to fly again until it is back within the defined region. There is more freedom of the third boundary for particles to roam around, and thus their natural motion would not be interfered. According to Robinson et al.

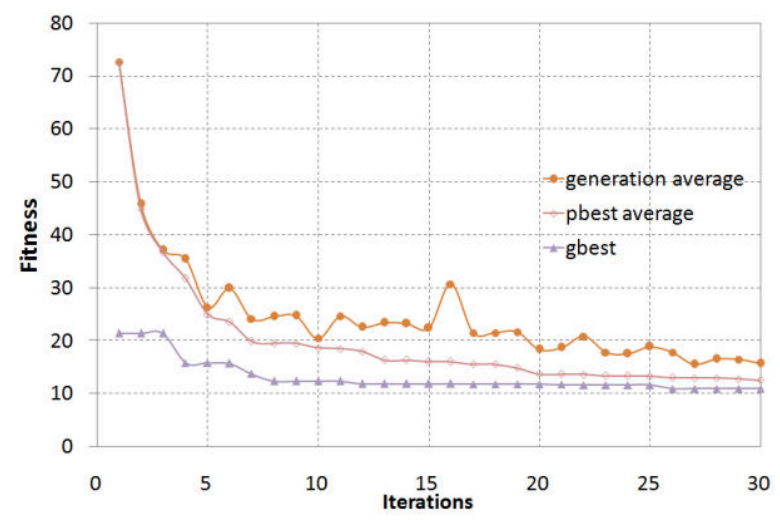

Fig. 3: Fitness of objective function against iteration.

in [11], invisible walls condition is faster and more consistent, and is thus adopted in this case.

\subsection{Objective function}

Each position of particles represents a possible design for the AFPM generator. To evaluate the goodness of the designs explored by the particles, an objective function needs to be defined and linked to return fitness values. In order to meet the required performance and at the same time reduce the cost, five performance indexes, namely weight of magnet $\left(M_{m}\right)$, weight of copper $\left(M_{c}\right)$, machine efficiency $(\eta)$, volume of the machine $\left(V_{g}\right)$ and power output $\left(P_{o}\right)$, are included in the objective function. The weight coefficients are contained before each performance index to indicate how much influence of each performance index has on the overall design. For the proposed machine, the objective function is defined as follow

fitness $=k_{m} \cdot M_{m}+k_{c} \cdot M_{c}+k_{v} \cdot V_{g}+k_{\eta} \cdot(1-\eta)-k_{p o} \cdot P_{o}$

where $k_{m}, k_{c}, k_{v}, k_{\eta}$, and $k_{p o}$ are corresponding weight coefficients. In general, different weight coefficient choices represent different design goals. In this case, performance and the cost of the generator are the key criterion for optimization. For instance, PMs are much more important and expensive than coils, and hence the value of $k_{m}$ is much larger than $k_{c}$. Furthermore, small volume, high efficiency and power output are preferred. Based on the design requirements and the range of variation, the weight coefficients are chosen as listed in Table 1, together with initial weight and scaling factors.

\subsection{Implement of PSO}

The flow chart of the PSO program is illustrated in Fig. 2. After initialization, the process of each iteration can be divided into 4 steps. Firstly, simulate and evaluate each position (solution) of the iteration. And then update $p_{\text {best }}$ and $g_{\text {best }}$. Thirdly, the velocities and the positions of next iteration are generated. At last, a judgement is carried out to make sure all particles are searching within solution space for the next iteration.

In this study, the fitness of objective function has been successfully converged after 30 iterations. The average fitness of particle positions, average fitness of personal best, and global best of each iteration is depicted in Fig. 3. It is shown that from $20^{\text {th }}$ iteration, the personal bests are already 


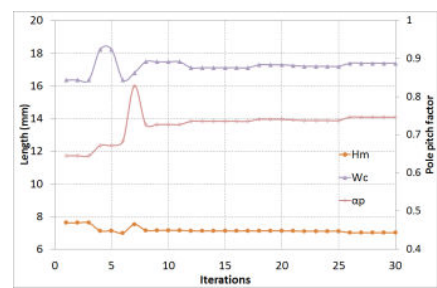

(a)

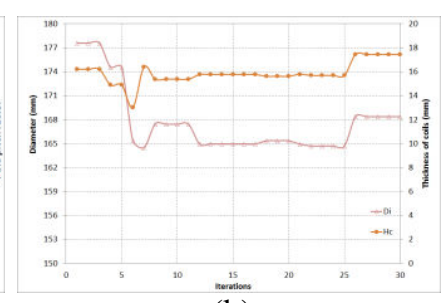

(b)
Fig. 4: Trends of best design parameters against iteration.

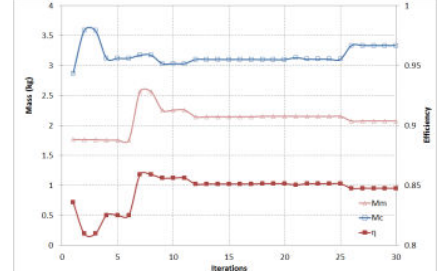

(a)

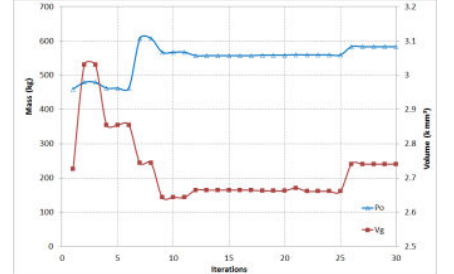

(b)
Fig. 5: Trends of performance indexes against iteration.

localized around their global best position in solution space. After 30 iterations good convergence is achieved, and the best solution found in iteration 26 , is taken as the optimal design. As shown in Fig. 4(a), after the first several iterations' fluctuation, the design parameters of $H_{m}, \alpha_{p}$, and $W_{c}$ find their corresponding optimal values, while $D_{i}$ has a great impact on the value of $H_{c}$ with large fluctuations, as shown in Fig. 4(b). It should be noted that all the design parameters stabilise after the $26^{\text {th }}$ iteration, with optimal solution obtained.

The trends of performance indexes are derived and depicted in Fig.5. As expected, usage of PM has higher influence to the evaluation. After an attempt to increase PM material, the usage is reduced gradually from $7^{\text {th }}$ iteration, while the usage of copper is increased to maintain the output. Though this brings out the issue of low efficiency and big in volume, the benefit of smaller amount of PM thus lower in cost and high power output makes the design a more practical choice.

\section{3-D FEA verification}

To validate the performance of the final optimised design from PSO algorithm, simulation should be carried out by using the FEA method. Because of the instinct structure of AFPM machines, 3-D FEA model is a used to obtain more accurate results. Since only back iron, PM poles and coils matter in the electromagnetic simulation, housing and connection parts of the machine are not included in the 3-D FEA model. The machine in 3-D model is illustrated in Fig.1, and the main design parameters are listed in Table 2.

\subsection{No load characteristics}

Firstly, the no-load characteristics of the machine are calculated. The flux distributions of the back iron and the air gap are shown in Fig. 6. As is seen from Fig. 6(a), the magnitude of flux density in the air gap is between 0.5-0.6 T, which is relatively low compared to machines with slotted cores. For the back iron, its flux density should be at the knee point of its B-H curve, which is about 2.0 T, to make full use of the material as well as avoiding saturation. The back EMF

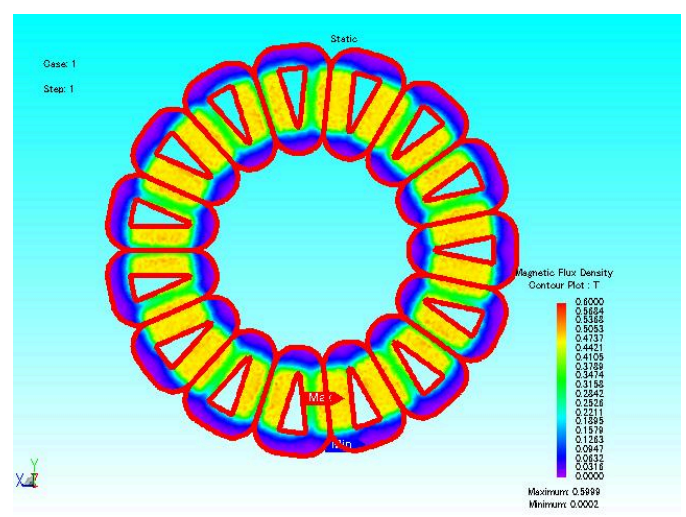

(a) air gap

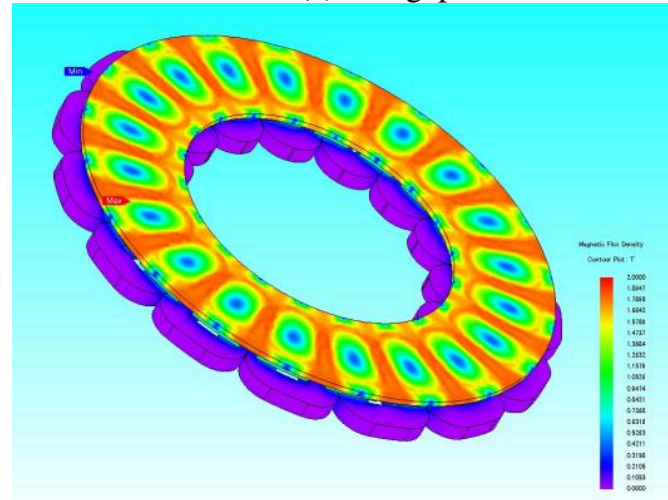

(b) back iron

Fig. 6: Flux distribution density in the machine

\begin{tabular}{|l|l|l|l|}
\hline Parameters & Value & Parameters & Value \\
\hline$P_{o}(\mathrm{~W})$ & 500 & $D_{o}(\mathrm{~mm})$ & 250 \\
\hline$n(\mathrm{rpm})$ & 300 & $D_{i}(\mathrm{~mm})$ & 168 \\
\hline$U_{o}(\mathrm{~V})$ & 220 & $H_{m}(\mathrm{~mm})$ & 7 \\
\hline$m$ & 3 & $\alpha_{p}$ & 0.75 \\
\hline$p$ & 20 & $H_{c}(\mathrm{~mm})$ & 17.4 \\
\hline$q$ & 15 & $W_{c}(\mathrm{~mm})$ & 16.8 \\
\hline
\end{tabular}

Table 2: Parameters of final design from PSO.

is another most important parameter for the machine design. For a three phase generator, sinusoidal wave form is preferred to avoid extra loss because of harmonic components. As is shown in Fig. 7(a), the back EMF wave form of the machine illustrates that the machine can achieve the output voltage requirement. Moreover, from Fig. 7(b), it can be seen that the output contains few harmonics. More specifically, the third harmonic is less than $2 \%$, which can be eliminated by using the star armature connection. For other higher harmonics, the magnitude of phase EMF is negligible. Therefore, such EMF is perfect for three-phase generator.

\subsection{On load characteristics}

To simulated performances of the generator at different operating points, FEA models at different loads are also constructed. For small-scale off-grid generator, loads can be classified into two categories, which are AC load for common household appliances and DC load for battery charging. To simulate the different conditions of these two loads, AC load and DC load are connected respectively to assure the output characteristics. 


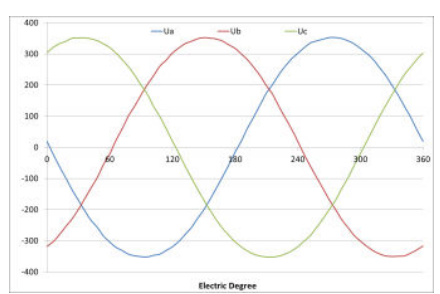

(a) back EMF waveform

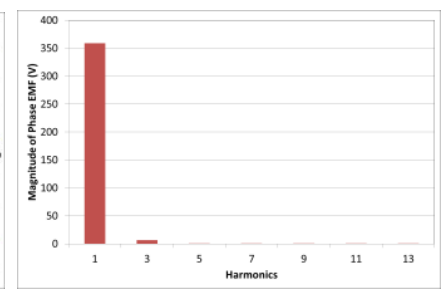

(b) back EMF spectrum

Fig. 7: Phase back EMF waveform and spectrum.

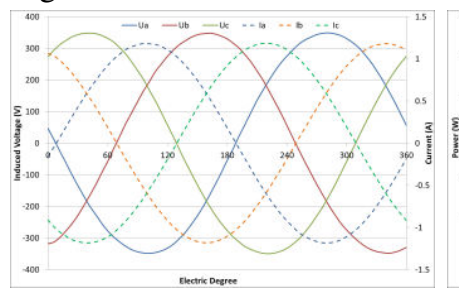

(a) phase voltage and current

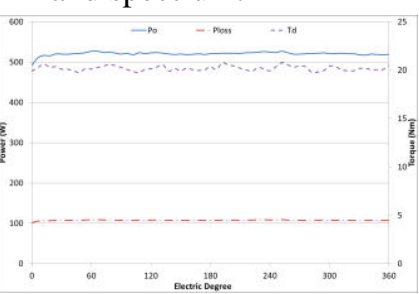

(b) loss, torque and power

Fig. 8: Output characteristics at AC load

Under the AC full load condition, both the induced voltage and armature current are quite sinusoidal, and the power output is over $520 \mathrm{~W}$ with a smooth torque, as depicted in Fig. 8. In fact, the loss is mainly caused by copper loss, because eddy current loss and core loss are eliminated due to the absence of the stator core. Also, because of the large airgap and thus low armature reaction, the iron loss in stator back iron is negligible. Since more copper conductors are used, $107 \mathrm{~W}$ is consumed by armature resistance. The resulting efficiency is $82.9 \%$.

Under DC load condition, a non-controllable three-phase rectifier is connected to obtain DC output. And because of the influence of the electronic switches during commutation, distortion can be observed in the voltage wave form, and thus output DC voltage is fluctuating six times in one electrical period in terms of the six switches of the three-phase fullwave rectifiers, as is shown in Fig. 9(a) and 9(b). In addition, from Fast Fourier transform (FFT) analysis of the DC voltage depicted in Fig. 9(c), it can be seen that there are only the 6th harmonic and the harmonics which are multiple of six except for DC component. Besides, greatly influenced by the fluctuation from voltage, the output power and magnetic torque also suffer from variations as shown in Fig. 9(d). The fluctuation is up to $20 \%$ of the average value. With the fluctuating currents, the copper loss is $9 \mathrm{~W}$ higher than that with DC load. Thus the efficiency at DC load is $81.2 \%$, which is lower than that under AC load. However, it should be noted that no filter or control algorithm is used in the simulation. In practical applications, power electronic circuit with controller and filters should be applied to smooth the output voltage and obtain a stable DC voltage. Due to the instability of wind speed, the wind turbine should be designed to adapt all these operating conditions at a wide wind-speed range. Thus, simulations are necessary to carried out at various load conditions to predict the output characteristics of the wind generator. Generator voltage regulation and efficiency at different load conditions with AC load and that with DC load are illustrated in Fig. 10. Generally speaking, the trends of these two figures are quite similar. In particular, it can be seen

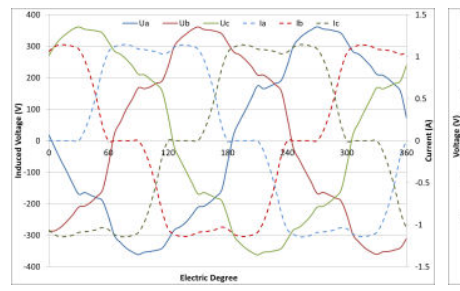

(a) phase voltage and current

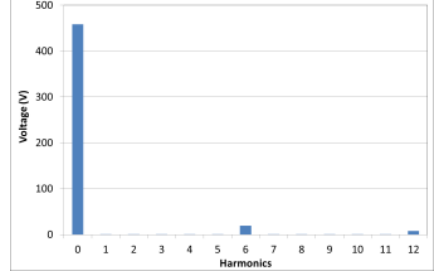

(c) DC voltage spectrum

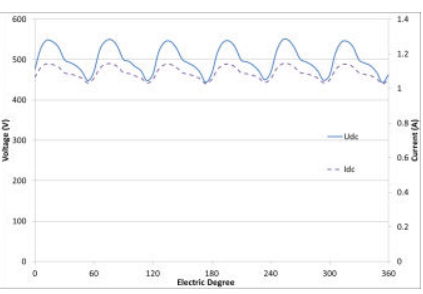

(b) DC voltage and current

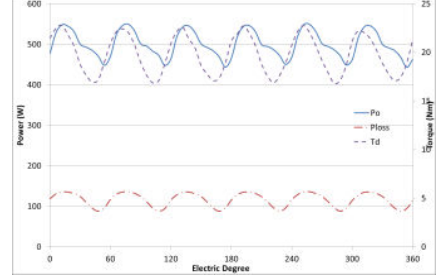

(d) loss, torque and power
Fig. 9: Output characteristics at DC load power output.

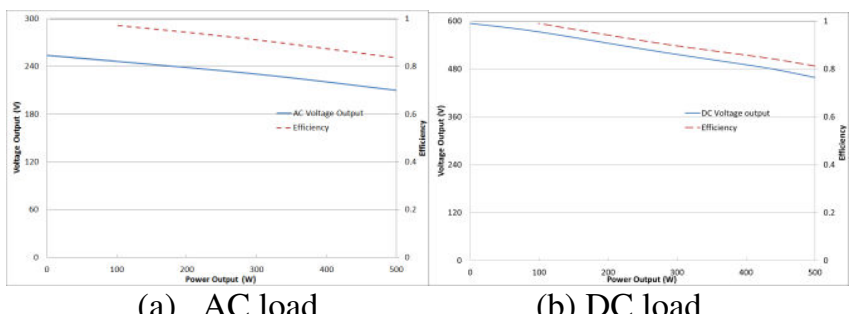

Fig. 10: Generator voltage regulation and efficiency at varied loads

that the operating efficiency is above $81 \%$, and the lowest efficiency appears at full load, which is because higher current leads to higher resistance loss. In addition, the output voltage and efficiency is linear against output power, which means that the generator can be treated as a linear system. Last but not least, for the small wind generator, the turbine is fixed and cannot be adjusted in terms of different wind speeds, which means that the energy captured by generator is changing all the time. In other words, it is impossible for the machine to operate at full load condition at all times. Therefore, the average operating point is probably at around $400 \mathrm{~W}$, and the average efficiency should be about $87 \%$.

In summary, according to PSO algorithm, a design with more copper and less PM is chosen as the optimal design. 3D FEA model is built and simulated under different load circumstances. Simulation result reveals that the final design has good performance with acceptable efficiency, and achieves the design requirements for small wind power systems. Furthermore, the usage of PM material for the machine is reduced with approximately same output features, which would minimize the cost of the machine.

\section{Conclusion}

In this paper, an AFPM synchronous machine with rated power of $500 \mathrm{~W}$ at $300 \mathrm{rpm}$ is proposed for small-scale wind power applications. A sophisticated multi-parameter and multi-objective PSO algorithm based on 3-D FEA is developed and employed to carry out optimization with a user defined objective function. The performance of the final optimal machine has also been comprehensively evaluated by 
the 3D FEA model. Results reveal that the optimal design from PSO has a good performance under different load conditions and meets the requirements as a small-scale directdrive wind power system, and PSO method is a simple and efficient optimization algorithm with fast convergence.

\section{Acknowledgements}

This work is associated with the iGIVE programme supported by the Engineering and Physics Science Research Council, U.K., Ref. EP/L001063/1.

\section{References}

[1] H. S. Kim and D. D.-C. Lu, "Review on wind turbine generators and power electronic converters with the grid-connection issues," Universities Power Engineering Conference (AUPEC), 2010 20th Australasian. pp. 1-6, (2010).

[2] L. Bin, Z. Jingyang, P. Yi, L. Qiang, Z. Haiming, and D. Qiang, "Review of Generation Schedule Methods with Large-Scale Wind Power Integration," Power and Energy Engineering Conference (APPEEC), 2011 AsiaPacific. pp. 1-4, (2011).

[3] M. Liserre, R. Cardenas, M. Molinas, and J. Rodriguez, "Overview of Multi-MW Wind Turbines and Wind Parks," Industrial Electronics, IEEE Transactions on, vol. 58, no. 4. pp. 1081-1095, (2011).

[4] D. Li, S. Wang, and P. Yuan, "A Review of Micro Wind Turbines in the Built Environment," Power and Energy Engineering Conference (APPEEC), 2010 Asia-Pacific. pp. 1-4, (2010).

[5] W. Liang and W. Liu, "Key technologies analysis of small scale non-grid-connected wind turbines: A review," World Non-Grid-Connected Wind Power and Energy Conference (WNWEC), 2010. pp. 1-6, (2010).

[6] T. F. Chan and L. L. Lai, "An axial-flux permanent magnet synchronous generator for a direct-coupled wind-turbine system", IEEE Transactions On Energy Conversion, vol. 22, No.1, pp. 86-94, (2007).

[7] N. Takorabet, J. P. Martin, F. Meibody-Tabar, F. Sharif, and P. Fontaine, "Design and optimization of a permanent magnet axial flux wheel motors for electric vehicle," Electrical Machines (ICEM), 2012 XXth International Conference on , pp.2635-2640, (2012).

[8] R. J. Hill-Cottingham, P.C. Coles, J.F. Eastham, F. Profumo, A. Tenconi, and G. Gianolio, "Multi-disc axial flux stratospheric aircraft propeller drive," IEEE Industry Applications Conference, 2001, vol.3, pp.16341639, (2001).

[9] H. M. Hasanien, "Particle Swarm Design Optimization of Transverse Flux Linear Motor for Weight Reduction and Improvement of Thrust Force," Industrial Electronics, IEEE Transactions on, vol. 58, no. 9. pp. 4048-4056, (2011).

[10] T. O. Ting, M. V. C. Rao, and C. K. Loo, "A novel approach for unit commitment problem via an effective hybrid particle swarm optimization," Power Systems, IEEE Transactions on, vol. 21, no. 1. pp. 411-418, (2006).

[11] J. Robinson and Y. Rahmat-Samii, "Particle swarm optimization in electromagnetics," Antennas and Propagation, IEEE Transactions on, vol. 52, no. 2. pp. 397-407, (2004).

[12] B. Zhao, C. X. Guo, and Y. J. Cao, "A multiagent-based particle swarm optimization approach for optimal reactive power dispatch," Power Systems, IEEE Transactions on, vol. 20, no. 2. pp. 1070-1078, (2005).

[13] R. C. Eberhart and Y. Shi, "Particle swarm optimization: developments, applications and resources," Evolutionary Computation, 2001. Proceedings of the 2001 Congress on, vol. 1. pp. 81-86 vol. 1, (2001).

[14] J. Kennedy and R. Eberhart, "Particle swarm optimization," Neural Networks, 1995. Proceedings., IEEE International Conference on, vol. 4. pp. 19421948, (1995).

[15] R. Eberhart and J. Kennedy, "A new optimizer using particle swarm theory," Micro Machine and Human Science, MHS '95., Proceedings of the Sixth International Symposium on. pp. 39-43, (1995).

[16] M. R. AlRashidi and M. E. El-Hawary, "A Survey of Particle Swarm Optimization Applications in Electric Power Systems," Evolutionary Computation, IEEE Transactions on, vol. 13, no. 4. pp. 913-918, (2009).

[17] [Z. Ren, X. Fang, S. Wang, J. Qiu, J. G. Zhu, Y. Guo, X. Yang, Z. Wang, Y. Sun, and J. Zhang, "Design optimization of an interior-type permanent magnet BLDC motor using PSO and improved MEC," Electrical Machines and Systems, 2007. ICEMS. International Conference on. pp. 1350-1353, 2007.

[18] Y. Duan, R. G. Harley, and T. G. Habetler, "A useful multi-objective optimization design method for PM motors considering nonlinear material properties," Energy Conversion Congress and Exposition. ECCE 2009. IEEE. pp. 187-193, (2009).

[19] G. Q. Bao, D. Zhang, J. H. Shi, and J. Z. Jiang, "Optimal design for cogging torque reduction of transverse flux permanent motor using particle swarm optimization algorithm," IPEMC 2004. vol. 1. pp. 260-263 Vol.1, (2004).

[20] A. A. Arkadan, M. N. ElBsat, and M. A. Mneimneh, "Particle Swarm Design Optimization of ALA Rotor SynRM for Traction Applications," Magnetics, IEEE Transactions on, vol. 45, no. 3. pp. 956-959, (2009).

[21] P. Guo, D. Huang, D. Feng, W. Yu, and H. Zhang, "Optimized design of induction motor parameters based on PSO (Particle Swarm Optimization)," Mechatronics and Automation (ICMA), 2012 International Conference on. pp. 837-842, (2012).

[22] T. K. Das and G. K. Venayagamoorthy, "Optimal design of power system stabilizers using a small population based PSO," Power Engineering Society General Meeting, 2006. IEEE. pp. 411-418, (2006). 